\title{
MONITORING THE FINAL ORBITAL DECAY AND THE RE-ENTRY OF TIANGONG-1 WITH THE ITALIAN SST GROUND SENSOR NETWORK
}

\author{
E. Vellutini ${ }^{\text {a }}$, G. Bianchi ${ }^{\text {b*, E. Perozzi }}{ }^{\text {a }}$, C. Pardini c ${ }^{\text {, L. Anselmo }}{ }^{\text {c }}$, T. Pisanu ${ }^{\text {d }}$ P. Di Lizia ${ }^{\text {e }}$ F. Piergentili ${ }^{\text {f }}$, \\ F. Monaci ${ }^{\text {g, M. Reali }}{ }^{\text {g, W. W. Villadei }}{ }^{\mathrm{g}}$, A. Buzzoni ${ }^{\text {h }}$, G. D'Amore ${ }^{\text {a }}$, L. Muolo ${ }^{\text {a }}$
}

\begin{abstract}
a Italian Space Agency (ASI), via del Politecnico snc, 00133 Rome, Italy elena.vellutini@est.asi.it, ettore.perozzi@asi.it, giuseppe.damore@asi.it

b INAF - IRA, Via Piero Gobetti, 101, 40129 Bologna, Italy, g.bianchi@ira.inaf.it

c ISTI-CNR, Via Giuseppe Moruzzi, 1, 56127 Pisa, Italy, carmen.pardini@isti.cnr.it, luciano.anselmo@isti.cnr.it

${ }^{\mathrm{d}}$ INAF - OAC, Via della Scienza, 5, 09047 Cuccuru Angius, Selargius, Cagliari, Italy, tpisanu@oa-cagliari.inaf.it

e Politecnico di Milano, Via Giuseppe La Masa, 34, 20156 Milano, Italy, pierluigi.dilizia@polimi.it

f Università La Sapienza, Via Eudossiana, 18, 00184 Roma, Italy, fabrizio.piergentili@uniroma1.it

${ }^{\mathrm{g}}$ Minister of Defence, Italian Air Force, Via Venti Settembre, 123/A, 00187 Roma, Italy,

fabio.monaci@aeronautica.difesa.it,marco.reali@aeronautica.difesa.it,walter.villadei@aeronautica.difesa.it

h INAF - OABO, Via Piero Gobetti, 93/3, 40129 Bologna, Italy, alberto.buzzoni@oabo.inaf.it

* Corresponding Author
\end{abstract}

\begin{abstract}
The uncontrolled re-entry of spacecraft and upper stages is quite common, occurring nearly every week. Among them, intact objects having a mass greater than five metric tons re-enter, on average, 1-2 times per year. Therefore, the re-entry of the first Chinese Space Station, Tiangong-1, was far from unusual, but attracted anyway a great worldwide attention and some concerns. For these reasons, the Italian component of the European SST (Space Surveillance and Tracking) consortium took this opportunity for carrying out a national exercise.

According to Chinese official sources, the ground control of Tiangong-1 was lost in March 2016, precluding the planned de-orbiting in the South Pacific Ocean Unpopulated Area (SPOUA). Tiangong-1 consisted of a cylindrical section, $10: 5 \mathrm{~m}$ in length and $3: 4 \mathrm{~m}$ in (maximum) diameter, with two rectangular solar panels of $3 \mathrm{~m} \times 7 \mathrm{~m}$. The mass was estimated to be around $7500 \mathrm{~kg}$.

The Italian network of sensors activated for the campaign included mono-static and bi-static radars, optical telescopes, a laser ranging station and a network of all-sky cameras, originally deployed for the observation of fireballs and bolides. In addition to providing complementary information, concerning the orbit, the attitude and the photometry of Tiangong-1, this quite heterogeneous collection of national assets provided also the occasion for testing, in an operational environment, the Italian sensor tasking preparedness and the data acquisition, exchange and processing capabilities within the European SST consortium.

In this respect, it is important to remember that in 2014 the European Commission, well aware of the topic criticality, took the commitment to implement a European network of sensors for surveillance and tracking of objects in Earth's orbit by starting a dedicated SST support framework program. Italy, France, Germany, Spain and UK joined it and constituted, together with SatCent, the front desk for SST services, the EUSST Consortium.

In this paper, a description of the Tiangong- 1 monitoring activities and of the main observations results obtained by the Italian sensor network are reported. Attention is also devoted to the coordination aspects of several Italian entities (military, civil and research organizations) that worked together. Finally, a description of the re-entry prediction and alert procedure for the national civil protection authorities is presented.
\end{abstract}

Keywords: Tiangong-1; Space Surveillance and Tracking; EUSST Consortium; Uncontrolled re-entries; Orbit determination; Re-entry predictions.

\section{Introduction}

The accurate prediction of re-entry impact points of uncontrolled space objects is currently a target not achievable for the physical uncertainty of the phenomenon (such as the solar flux forecast, the total mass and aerodynamics of the space object), but also for errors and irregularity of tracking data.

JSpOC provides for decaying objects updated TLE (Two Line Elements) while TIP (Tracking and Impact
Prediction) messages for the estimation of the impact point location and time are released on space-track.org 4 days, 3 days, 2 days, 1 day, 12 hours, 6 hours and 2 hours before the estimated re-entry plus a final post reentry TIP message after the re-entry.

European Commission decided to cope with the risks related to space objects with the decision N. 541 of April 16th 2014 of European Parliament and of the Council [1], where a Framework for Space Surveillance and Tracking Support is established. As declared in 
Article 3 of the Decision, one of the specific objectives of this Framework is "1. The general objective of the SST Support Framework is to contribute to ensuring the long-term availability of European and national space infrastructure, facilities and services which are essential for the safety and security of the economies, societies and citizens in Europe. 2. The specific objectives of the SST support framework are: (a) assessing and reducing the risks to in-orbit operations of European spacecraft relating to collisions and enabling spacecraft operators to plan and carry out mitigation measures more efficiently; (b) reducing the risks relating to the launch of European spacecraft; (c) surveying uncontrolled re-entries of spacecraft or space debris into the Earth's atmosphere and providing more accurate and efficient early warnings with the aim of reducing the potential risks to the safety of Union citizens and mitigating potential damage to terrestrial infrastructure; (d) seeking to prevent the proliferation of space debris".

In this framework five European Member States, i.e. France, Germany, Italy, Spain and UK, have realized a partnership with the European Union, creating the EUSST Consortium. Each Member contributes to EUSST capability of surveillance and tracking of space objects with its national assets. Italy contributes with several sensors: optics, radar and laser.

Within this context, several Italian institutions cooperate and make available their specific competences for EUSST: the Italian Space Agency works as National Entity and provides its telescope SPADE and the MLRO laser located in Matera Center for Space Geodesy. The Minister of Defense (MoD) operates as National Operational Center (NOC) and has available telescopes and radars. The National Institute for Astrophysics (INAF) operates both passive optics and radars instruments. ASI, MoD and INAF have created in this specific scenario the Coordination and Steering Committee related to Space Surveillance and Tracking Initiative of European Commission OCIS (Organismo di Coordinamento e di Indirizzo relativo all'iniziativa Space Surveillance and Tracking della Commissione Europea) in order to coordinate Italian capabilities and quickly respond to high interest events such as Tiangong-1 re-entry.

For this specific event, the OCIS identified two additional Italian entities useful for the activities of monitoring and re-entry prediction. In particular, The University of Roma "La Sapienza" and the ISTI-CNR institute have been involved for their competences in monitoring and light curve analysis for asset determination and reentry predictions for Civil Protection purposes.

Even if the uncontrolled re-entry of objects from space is a rather frequent event, Tiangong-1 re-entry raised some concerns since its re-entry footprint might intersect the Italian territory and because some hydrazine could left over, resulting in an explosion with possible pollution on ground and in sizable macroscopic fragments.

According to Chinese official sources, since March 2016 Tiangong-1 was no longer responding to ground control, precluding the planned de-orbiting in the South Pacific Ocean Unpopulated Area (SPOUA). Tiangong-1 consisted of a cylindrical section, $10.5 \mathrm{~m}$ in length and $3.4 \mathrm{~m}$ in (maximum) diameter, with two rectangular solar panels of $3 \mathrm{mx} 7 \mathrm{~m}$. The mass was estimated to be around $7500 \mathrm{~kg}$.

For the first time since its establishment, for the Tiangong-1 re-entry, OCIS worked for an operative campaign, by coordinating all the Italian actors involved, tasking the Italian sensors employed to monitor the orbital decay, providing re-entry predictions and supporting Civil Protection Department during the final stages of the re-entry.

In the following paragraphs the Italian network activated for the Tiangong-1 re-entry campaign will be descripted, as well as the operational results obtained both in terms of products such as orbit determination, TLE generation, re-entry predictions and coordination aspects between civil and military entities.

\section{Italian SST ground sensor network}

For the Tiangong- 1 re-entry campaign, several sensors have been employed both from the Italian EUSST architecture and by employing sensors of partner institutions such as La Sapienza. The sensors employed were laser:

- MLRO: Matera Laser Ranging Observatory; Optical:

- $\quad$ SPADE

- PdM Mite

- Sapienza Scientific Observatory Network

Radar:

- BIRALES

- BIRALET

- MFDR-MR

ASI contributes to the campaign with its laser MLRO and its telescope SPADE, both located in Matera Centre of Space Geodesy. MLRO (Matera Lasera Ranging Observatory) currently sends data to the International Laser Ranging Services (ILRS) and the Italian Operational Centre receives and uses the overall information from ILRS, but not specifically from this laser. For EUSST it is used for specific tracking requests of cooperative targets. During Tiangong-1 reentry campaign, the Chinese station was put in the scheduler and one acquisition was done. This has been an interesting result if one consider that the station was uncontrolled and, consequently, the precise position of the retroreflectors was unknown. 
SPADE telescope (Figure 1) has an aperture of 40 $\mathrm{cm}$ and a FoV of $1.38^{\circ} \times 1.38^{\circ}$. It is used $100 \%$ of the time for EUSST purposes but in an experimental mode. It surveys the GEO ring 3 or 4 times a night, with about 5 seconds exposure time. It is being used mainly in surveillance mode and its data is sent to ISOC. For Tiangong- 1 campaign it was also tasked for the station tracking, acquiring some images during the final stages of the re-entry.

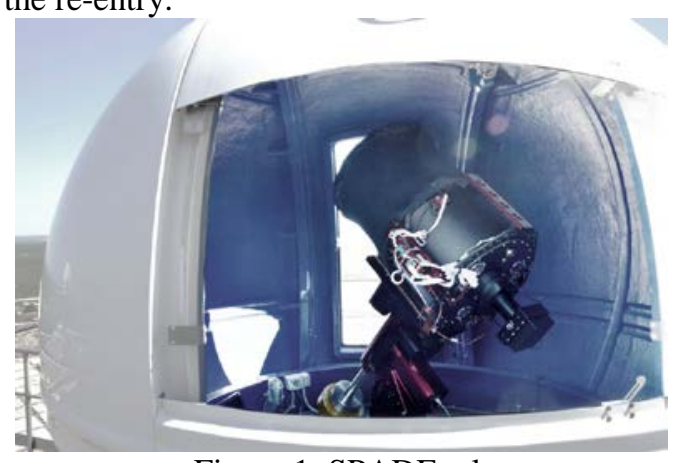

Figure 1. SPADE telescope

The Sapienza Scientific Observatory Network, managed by Sapienza University of Rome, has taken part in the monitoring campaign by performing optical observations. These have been used to retrieve both position angular measurements and light curves for attitude reconstruction of Tiangong- 1 space station. The employed optical sensors, which are MITO, RESDOS, SCUDO and EQUO OG observatories are located in Italy and Kenya. Such a distributed geometrical configuration ensures an increased number of visible passages. All telescopes have been operated by using a sidereal tracking observation strategy.

MITO telescope (Figure 2) is located in Rome, Italy $(41.95588 \mathrm{~N}, 12.50559 \mathrm{E}, 76 \mathrm{~m})$. It has a diameter of $25.0 \mathrm{~cm}$ and field of view of $3.5^{\circ} \times 2.5^{\circ}$.

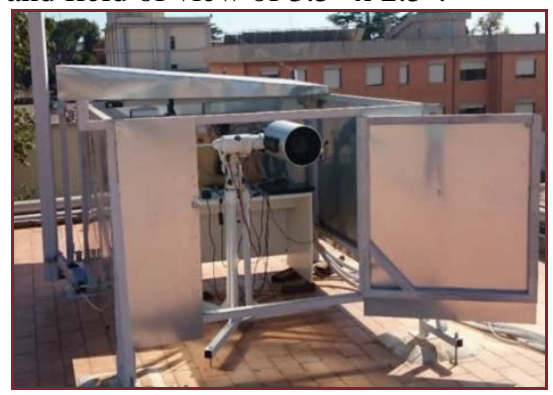

Figure 2. MITO telescope

RESDOS (Figure 3) is located in Avezzano, Italy $(42.06388 \mathrm{~N}, 13.43361 \mathrm{E}, 800 \mathrm{~m})$. It has a diameter of $40.0 \mathrm{~cm}$ and field of view of $2^{\circ} \times 2^{\circ}$.

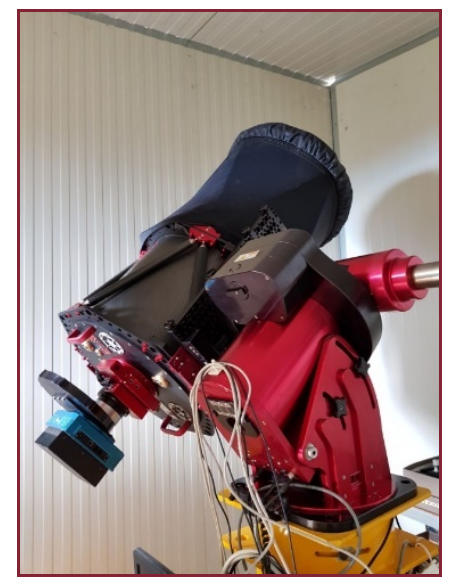

Figure 3. RESDOS telescope

SCUDO (Figure 4) is located in Collepardo, Italy $(41.76527 \mathrm{~N}, 13.37500 \mathrm{E}, 555 \mathrm{~m})$. It has a diameter of $25.0 \mathrm{~cm}$ and a field of view of $2.2^{\circ} \times 2.2^{\circ}$.

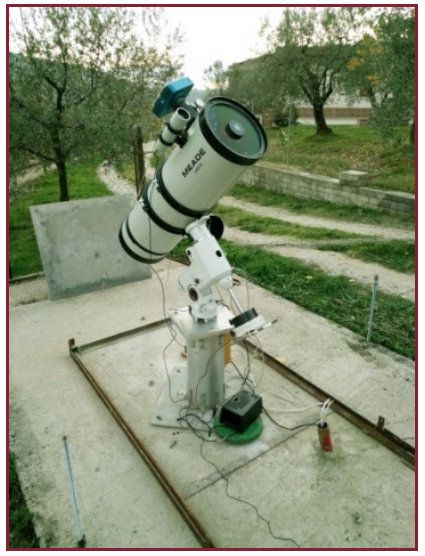

Figure 4. SCUDO telescope

EQUO-OG (Figure 5) is located in Malindi, Kenya (2.996373 S, $40.193791 \mathrm{E}, 0 \mathrm{~m}$ ). It has a diameter of $25.0 \mathrm{~cm}$ and a field of view of $2.2^{\circ} \times 2.2^{\circ}$.

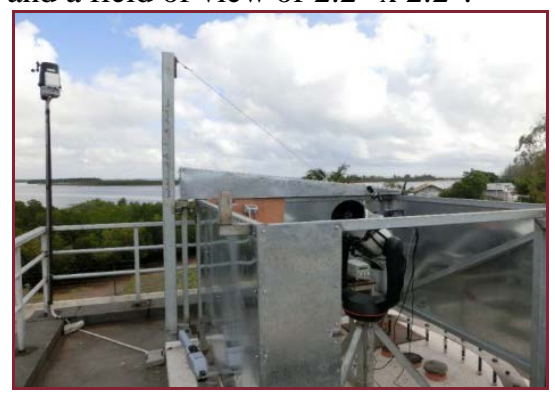

Figure 5. EQUO-OG telescope

INAF provided two radars for Tiangong-1 re-entry campaign: BIRALES and BIRALET. BIRALES is a bistatic radar composed of two distinct antennas, a receiving and a transmitting one with a baseline of about $580 \mathrm{~km}$. The transmitting antenna is the Radio Frequency Transmitter (TRF) of the Italian Joint Test Range of Salto di Quirra (PISQ) in Sardinia. It consists of a powerful amplifier able to supply a maximum power of $10 \mathrm{~kW}$ in the bandwidth $410-415 \mathrm{MHz}$. It is a $7 \mathrm{~m}$ dish completely steerable at a maximum speed of 3 
$\mathrm{deg} / \mathrm{sec}$ and with right hand circular polarization. It is available for operation $24 \mathrm{~h} /$ day. Its field of view (FoV) matches almost perfectly the receiving antenna one, with a beam of 6 deg.

The receiving antenna is a portion of the Northern Cross Radio Telescope (in Figure 6), which is currently one of the largest UHF-capable antenna in the world, being located at the Medicina Radio Astronomical Station, near Bologna, in Northern Italy. It is owned by the University of Bologna, but managed and operated by the Istituto di Radioastronomia at the Istituto Nazionale di Astrofisica (INAF-IRA). The portion dedicated for the BIRALES receiving antenna is actually composed of 16 parabolic cylindrical antennas of the N/S branch. The total collecting area is about 2800 square meters and it allows to detect small objects with sub-metric RCS (Radar Cross Section) at $1000 \mathrm{~km}$ of altitude.

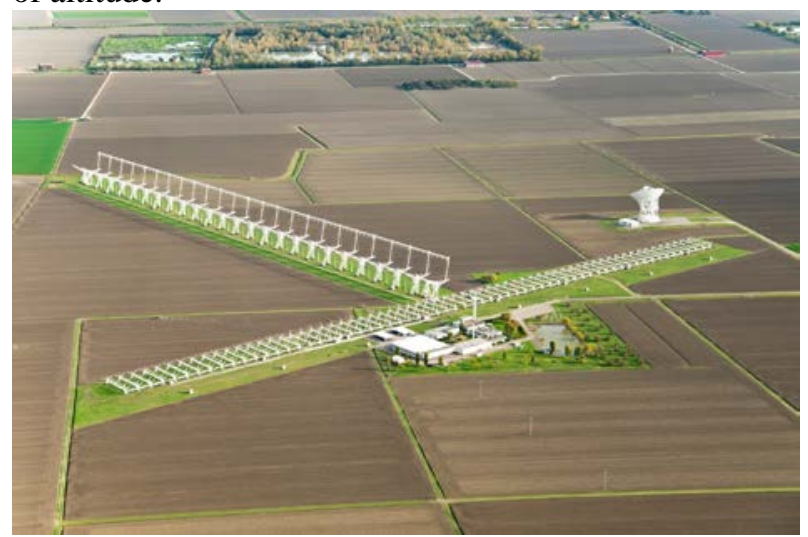

Figure 6. Northern Cross

BIRALES works in survey mode and exploits an innovative concept based on multi-beam technique. Indeed, due to the large numbers of receivers installed on the Northern Cross, the Field of View (FoV) can be populated with many independent beams. When an object transits inside the antenna FoV, beams are illuminated by the reflected radio waves. Thus, by looking at the beam illumination sequence, it is possible to estimate the ground track of the transiting objects, with a higher level of details with respect to a singlebeam system. The information about the sequence of illuminated beams allows to discern the trajectory of the object. In parallel, the TRF emits also a chirp of known period and span, in order to estimate the range; in this way BIRALES acquires all the information to estimate the target's orbit with a precision below $100 \mathrm{~m}$.

BIRALET (Bistatic Radar for Leo Tracking) is a bistatic radar which uses the same transmitting antenna as BIRALES, but the receiving antenna is the SRT (Sardinia Radio Telescope in Figure 7): a 64 meters wheel-and-track, fully steerable parabolic antenna with a Gregorian optical configuration. The SRT is designed to observe the sky with high efficiency in the frequency range between $300 \mathrm{MHz}$ and $116 \mathrm{GHz}$ and is able to host up to 20 remotely controllable receivers. In order to observe the signal transmitted from the TRF and scattered by the debris, the coaxial dual-feed L-P band $(0.305-0.410 \mathrm{GHz}, 1.3-1.8 \mathrm{GHz})$ cryogenic receiver of SRT has been used. BIRALET can work in beam parking or tracking mode, it has a gain of $47 \mathrm{~dB}$, a 3dBbeamwidth of 0.8 degrees, a receiver noise temperature of about 20 Kelvin, a physical area of more than 3200 square meters. Now BIRALET can provide only the Doppler measurements of the observed debris but we are planning the upgrade to the range measurement too.

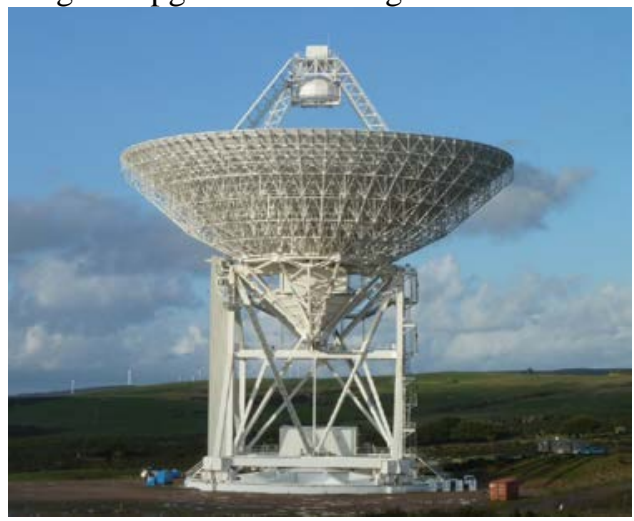

Figure 7. Sardinia Radio telescope

Italian MoD contributed for this campaign with passive optics (PdM-MiTe in Figure 8) and radar instruments (MFDR in Figure 9). PdM-MiTe is an equatorial mount telescope with a diameter of $350 \mathrm{~mm}$, a focal distance of $980 \mathrm{~mm}$ and a focal ratio of 2,8. It can rely on GPS timing and weather sensors station.

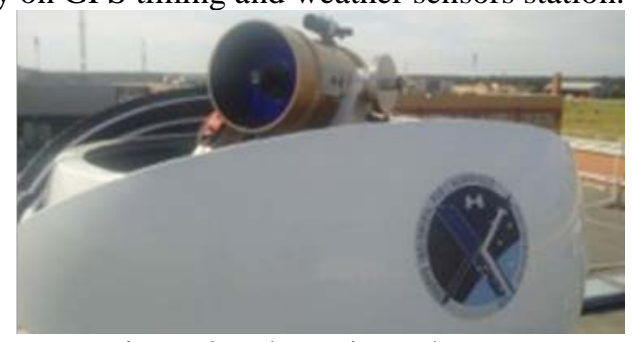

Figure 8. PdM-MiTe telescope

Weibel MFDR-MR is a Phased-Array Doppler radar with a $42 \mathrm{~dB}$ antenna and a transmission power of 320 $\mathrm{W}$. The radar is a continuous-wave (CW) system and through the transmission of Multi Frequency (MF) waves or with frequency modulation (FM) is able to perform the tracking and ranging of orbital objects.

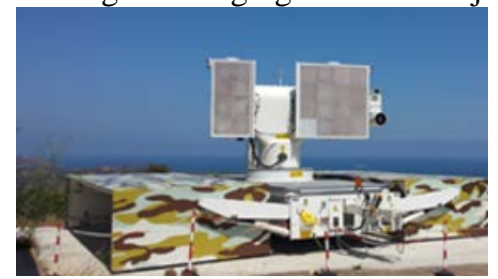

Figure 9. MFDR 


\section{Tiangong-1 test campaign: operational results}

ASI and MoD coordinated the activities among the actors involved in the re-entry campaign. In particular, ASI was the interface with the Italian Civil Protection department and was in charge of the communication with the media, while the MoD, with Italian SST Operational Centre ISOC, was in charge of the overall operational process.

\subsection{Workflow for Tiangong-1 re-entry}

Considering the subjects involved in the experimental Campaign, a set of relationships among them was established and specific tasks and responsibilities assigned to each of them. In particular, ASI played the role of front-end towards the Department of Civil Protection. Behind ASI, an articulated technical, scientific and operational machine was put in place, mainly based on CNR-ISTI, the technical-scientific reference for re-entry analysis, and ISOC, the national operational center, deputed to the provision of SST services both for national and European usage. ISOC was linked to both national sensors and external Entities for information and sensor data exchange, necessary to support both the national activities on the Tiangon-1 Re-entry monitoring and to provide other players (EUSST and US JSpOC) with the national contribution to their respective activities.

The articulated set of relations among each single national and external subject is summarized in the scheme reported below (Figure 10).

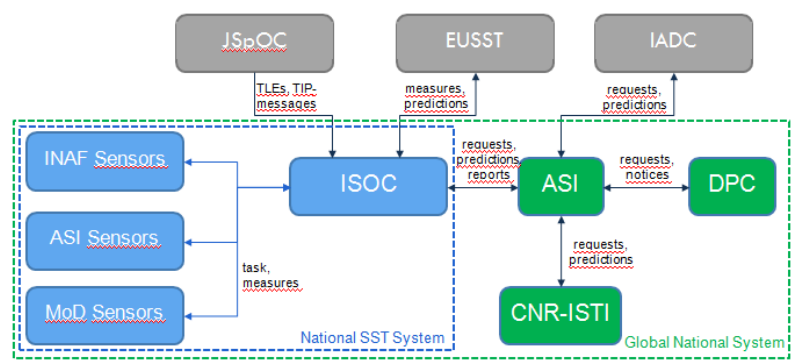

Figure 10. Overall Organizational Scheme

ASI received updated predictions and products (mainly reports) from both CNR-ISTI and ISOC, in order to update the Technical Board at the Department of Civil Protection with timely and reliable notices about the evolution of the event.

Within the National SST System perimeter, ISOC was in charge to orchestrate the overall operational process, consisting of three main phases:

1. Sensor planning/tasking;

2. Data processing;

3. Product output and delivery.

The sensor planning/tasking was simplified, due to the fact that the re-entering object was known to all the sensor authorities and highest priority was assigned to this task. So this phase consisted essentially of sensor tasking at an appropriate pace, as agreed in the specific operational regime (see in the following). Sensor tasking was possible using TLEs from the US JSpOC, other EUSST Partners and auto-generated. An acknowledgment was expected by each sensor authority after the receipt of a task, in order to confirm the feasibility of the task itself.

After the sensor operation, the data/measurement obtained were delivered to ISOC, by means of a VPN/ftp connection established within the ISOCsensors network. In case measurement were from sensors contributing to other campaigns (i.e. the US JSpOC Campaign and EUSST Campaign), they were also delivered to the respective recipients (i.e. respectively, US JSpOC and S3TOC).

In the second phase, using the sensor data available, ISOC could compute the updated object orbit and propagate it in order to predict its re-entry epoch, by means of both AGI STK-based and auto-generated tools; the re-entry site prediction was reasonably processed only during the day before the re-entry, once the last orbits were steady.

Finally, in the third phase, products in support of both the national campaign (reports) and the EUSST campaign (predictions) were output and delivered to the respective recipients (i.e. respectively, ASI and S3TOC).

Within the national framework, JSpOC was exclusively in contact with ISOC during the whole campaign, by means of a Liaison Officer from the Italian Air Force. JSpOC provided ISOC with TLEs, used both for sensor tasking and reference in the orbital analysis, predictions and TIP messages, used both as a reference and to rule the operational regime transition. Precision tasking was possible by means of autogenerated TLEs and TLEs from EUSST Partners, during the last days/hours before the re-entry.

Different operational regimes (Figure 11) were setup, in order to apply an operational effort proportional to the severity/imminence of the event. They were tailored on JSpOC operational regimes.

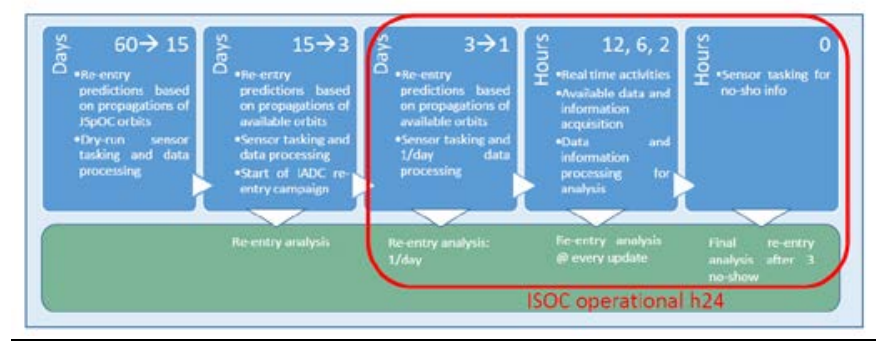

Figure 11. ISOC Operational Regimes for RE Service

In accordance to the above-reported scheme, ISOC was operational h24 during the last three days before the object re-entry. 


\subsection{Orbit determination}

Orbit determination was performed by two separate entities independently: ISOC by employing the data received by Italian sensors involved and Politecnico di Milano for BIRALES observations.

ISOC Team used AGI Orbit Determination Tool Kit (ODTK) to analyse the data received and processed in order to establish the goodness of the data as can be seen in the picture below where residuals of MFDR data are shown.

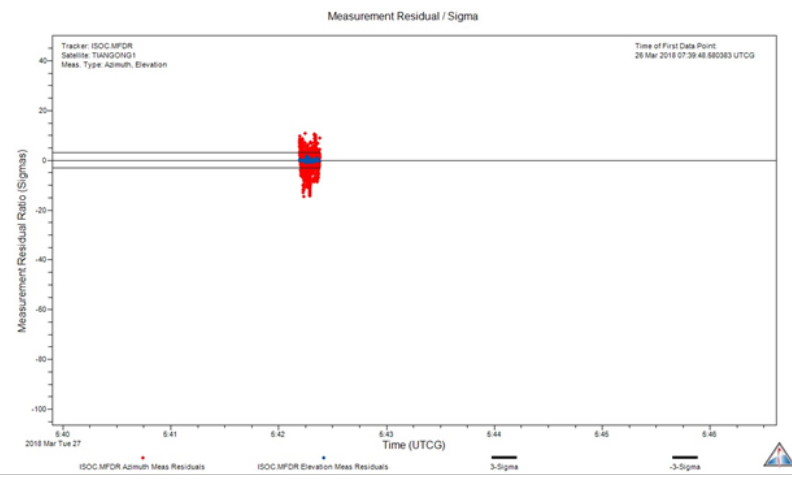

Figure 12. Residual ratio example

By means of data fusion it was possible to obtain a propagated orbit of the Chinese station and derive internal TLEs that the operation center used to task sensors as well.

Taking as reference the JSpOC TLEs, the following picture depicts the range between the propagated TLEs and the ISOC predicted orbit.

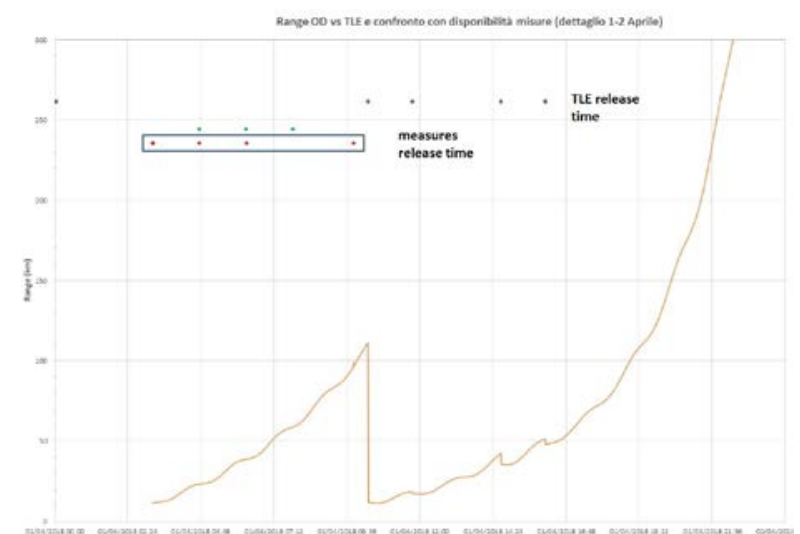

Figure 13. Range trend between ISOC OD and TLEs

As can be seen, once new TLEs were released, the range drops down indicating that the ISOC orbit determination (OD) was predicted in accordance with the orbit updated by JSpOC. The rectangle in the picture enclose the measures received and considered for the OD in the graph.
The deviation over time comes both from the SGP4 propagator used for TLEs that does not fit properly for a re-entering object, whereas the HPOP propagator used in the OD can better fit those kind of phenomena, and from the lack of measures from sensors.

The orbit determination (OD) of Tiangong-1 with the BIRALES sensor was based on the data gathered during two passages occurred on March 29 and March 31 (see Table 1 for details about the meridian crossing on BIRALES receiver). In the followings, the discussion is focused on the data and results obtained for the passage of March 29.

Table 1. Azimuth $\left(A z_{R X}\right)$ and elevation $\left(E l_{R X}\right)$ of Tiangong-1 at meridian crossing on BIRALES receiver

\begin{tabular}{lll}
\hline Epoch (UTC) & $\begin{array}{l}\text { AzRX } \\
\text { (deg) }\end{array}$ & $\begin{array}{l}\mathrm{El}_{\mathrm{RX}} \\
(\mathrm{deg})\end{array}$ \\
\hline 29 MAR 2018 07:56:03.27 & 180.00 & 40.83 \\
31 MAR 2018 06:54:32.55 & 180.00 & 39.05 \\
\hline
\end{tabular}

The measurements provided by the sensor were limited to the signal-to-noise ratio (SNR) and Doppler Shift (DS) profiles. No bistatic slant range measurement was acquired. Figure 14 and Figure 15 report the SNR and DS profiles as a function of the time difference between the measurement epoch and the meridian crossing epoch as predicted from the last TLE available before the passage. While passing through the Field-ofView (FoV) of BIRALES, Tiangong-1 simultaneously illuminates the main lobes and side lobes of the different beams. 


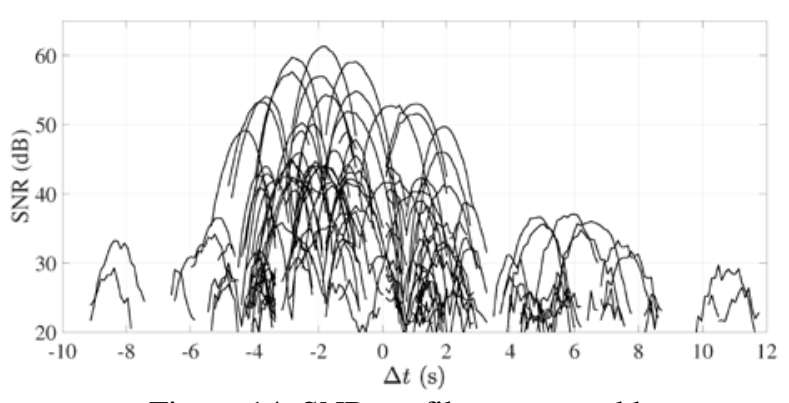

Figure 14. SNR profiles measured by BIRALES.

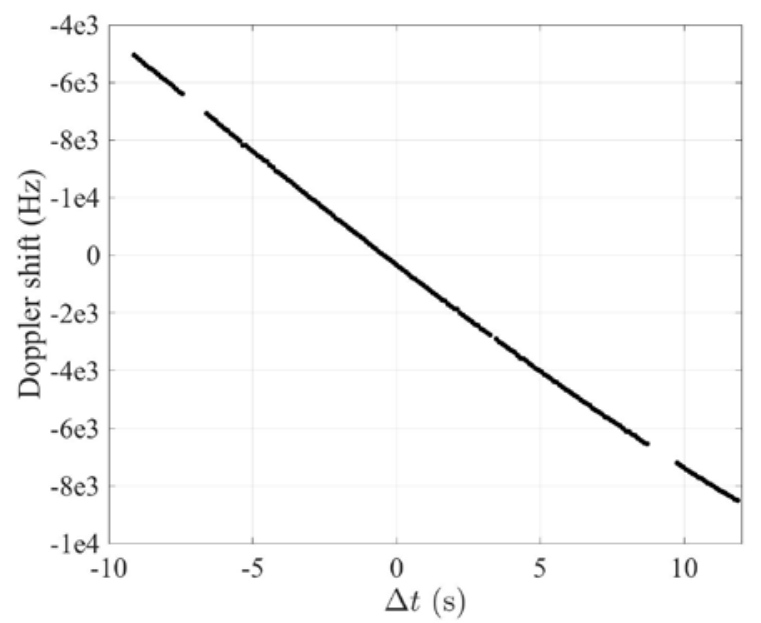

Figure 15. DS profile measured by BIRALES.

In addition, Figure 14 reveals a time delay of about two seconds between the meridian crossing epoch expected from the TLE and the actual crossing measured by the sensor, which can be linked to the orbital drift caused by the atmospheric drag acting on the space station.

In the absence of slant range measurements, the standard OD process adopted for BIRALES was properly modified. The OD was performed in two phases. The first phase was devoted to estimate the angular path of the object inside the FoV of the sensor based on the SNR profiles. To this aim, the angular path was approximated to be linear in time during the passage; i.e., the E-W $\left(\Delta \gamma_{1}\right)$ and N-S $\left(\Delta \gamma_{2}\right)$ angular displacements with respect to the receiver boresight were regressed as:

$$
\left\{\begin{array}{l}
\Delta \gamma_{1}=a_{1} t+a_{0} \\
\Delta \gamma_{2}=b_{1} t+b_{0}
\end{array}\right.
$$

The aim of the first phase was estimating the values of the coefficients of the regression. This goal was achieved by picking the SNR profiles of the beams crossed by Tiangong- 1 during the passage, based on the available TLE. The peaks of such profiles were then used within a linear fitting process to estimate the coefficients of the regression. The result of the first phase is reported in Figure 16, which compares the estimated angular path and the angular profiles predicted from the TLE for the same epochs. As can be seen, the comparison confirms the time delay between the measured and predicted profiles.

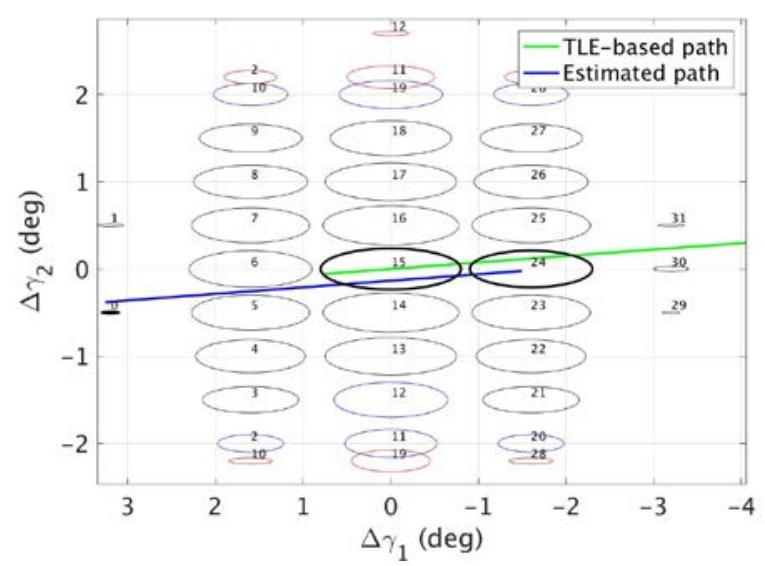

Figure 16. Comparison between the estimated angular path and the one predicted from the TLE.

The estimated angular path is then used in the second phase of the OD process. Here, the orbital state of Tiangong- 1 was estimated by minimizing the residuals on the SNR and angular profiles by adopting a least-squares method. Table 2 reports both the estimated orbital elements and the ones predicted using the available TLE. Figure 17 compares the ground track obtained by propagating the state derived in the OD process with the TLE-based prediction for one revolution after the passage.

Table 2. Orbital parameters comparison between TLE and OD.

\begin{tabular}{lll}
\hline Parameter & From & From OD \\
& TLE & \\
\hline Semimajor axis (km) & 6567.27 & 6558.75 \\
Eccentricity & 0.0012 & 0.0028 \\
Inclination (deg) & 42.655 & 42.661 \\
$R A A N$ (deg) & 218.04 & 217.96 \\
Argument of periapsis (deg) & 13.01 & 341.05 \\
True anomaly (deg) & 83.54 & 115.67 \\
\hline
\end{tabular}




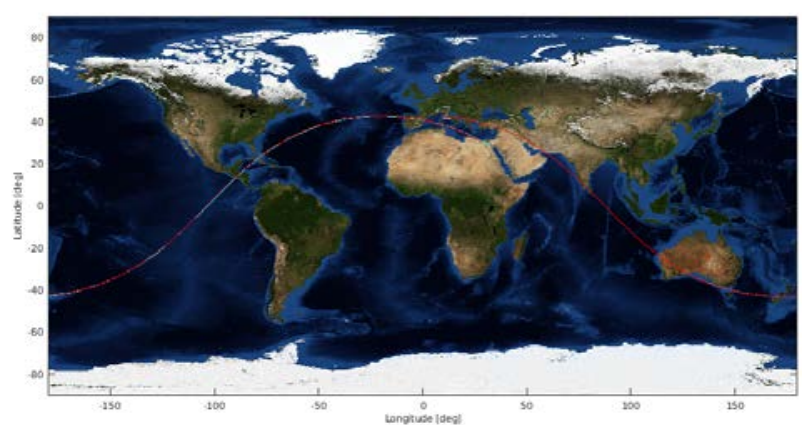

Figure 17. Comparison between the ground tracks of the determined orbit (blue line) and the TLE-based prediction (red line).

\subsection{Improvement of TLE accuracy}

By exploiting the optical measurements performed during the campaign, different strategies for the improvement of TLE accuracy have been tested. In particular, an orbit determination algorithm developed by Sapienza University, which performs a constrained optimization in the space of Kozai-Brouwer mean orbital parameters and $B^{*}$ to return a solution in the TLE format, has been run and tested with different state vector configurations. The solution has been consequently propagated to the time of subsequent measurements to compute residuals, and hence tested to assess the actual accuracy improvement. The analysis has been devoted to assessing which orbital parameters could be used as state vector for the optimization when the number and distribution of measurements are not sufficient to estimate all TLE parameters. In fact, this situation is very common when tracking re-entering objects. The following results have been obtained for the cases in which additional measurements were available in a 1-day interval after the observations used for the orbit determination. Different state vectors, corresponding to different combinations of orbital parameters, have been tested. In particular, a comparison has been performed between the results obtained through the seven TLE parameters estimation, through the estimation of 6 orbital parameters excluding $B^{*}$ and through the estimation of single parameters.

Table 3 shows the results obtained by performing the optimization using 6 measurements taken by MITO during 1 passage on February 15, 2018 from 04:59:00 UTC to 05:01:01 UTC. The epoch of the employed TLE is February 15, 2018 at 13:56:50 UTC.

The pre- and post-optimization angular residuals with respect to 2 measurements performed by MITO on February 16, 2018 at 04:53:59 UTC and at 04:54:01 UTC and 2 measurements performed by RESDOS on February 16, 2018 at 04:54:30 UTC and at 04:55:00 UTC, are compared for the different configurations.
Table 3. Mean angular residuals with respect to measurements taken by MITO and RESDOS on February 16, 2018, not used for the orbit determination

\begin{tabular}{|c|c|c|}
\hline State vector & MITO & RESDOS \\
\hline$\left[i, \Omega_{v} \theta_{v} \omega_{v} M_{v} n_{v} B^{*}\right]$ & $3.59 \times 10^{5}$ " & $2.01 \times 10^{5} "$ \\
\hline$\left[i, \Omega_{s} e_{s} \omega_{s} M_{s} n\right]$ & $3.56 \times 10^{5}$ " & $1.99 \times 10^{5}$ " \\
\hline [i] & 2040.97” & 1433.31" \\
\hline$[\Omega]$ & 1503.43” & 1034.37” \\
\hline [e] & 1500.50” & $966.28 ”$ \\
\hline$[\omega]$ & 1407.56” & 970.46” \\
\hline$[M]$ & 1406.44” & 970.33” \\
\hline [n] & 3164.07” & 2251.13” \\
\hline$\left[B^{*}\right]$ & $304.90 ”$ & 221.80" \\
\hline Initial Guess & 2060.85” & 1440.59” \\
\hline
\end{tabular}

Table 4 shows the results obtained by performing the optimization using 9 measurements taken by MITO during 1 passage on March 9, 2018 from 18:16:00 UTC to 18:18:00 UTC. The epoch of the employed TLE is March 9, 2018 at 13:22:43 UTC.

The pre- and post-optimization angular residuals with respect to 4 measurements performed by MITO on March 10, 2018 from 18:05:30 UTC to 18:06:30 UTC are compared for the different configurations.

Table 4. Mean angular residuals with respect to measurements taken by MITO on March 10, 2018, not used for the orbit determination

\begin{tabular}{ll}
\hline State vector & MITO \\
\hline$\left[i, \Omega, e_{s}, \omega_{,}, n_{s} B^{*}\right]$ & $2.61 \times 10^{5}$, \\
{$\left[i, \Omega, e_{w}, M, n\right]$} & $2.98 \times 10^{5}$, \\
{$[i]$} & $4907.16 ”$ \\
{$[\Omega]$} & $4528.25 ”$ \\
{$[e]$} & $4700.78 ”$ \\
{$[\omega]$} & $4388.46 ”$ \\
{$[M]$} & $4390.42 ”$ \\
{$[n]$} & $919.79 ”$ \\
{$\left[B^{*}\right]$} & $22636.24 ”$ \\
\hline Initial Guess & $5069.62 ”$ \\
\hline
\end{tabular}

The analysis has shown that, when a small number of measurements is available, the estimation of single orbital parameters could lead to an actual improvement of the state accuracy. In particular, while both the mean motion and the $B^{*}$ have not implied an improvement in both test cases, the other parameters have led to smaller residuals in all cases. Among these, the mean anomaly and the argument of perigee have led to the best results. In the second test case, the best results have been achieved by estimating the mean motion only. Further investigations will clarify whether the estimation of this parameter only con lead to the best state improvement as the object gets close to re-entry. 
The data produced during the observation campaign conducted for the re-entry phase of Tiangong 1 space station have been also exploited for the validation of a new algorithm, elaborated from Sapienza S5Lab, finalized to the identification of a space object position in terms of its height and distance from a specific ground station.

The algorithm exploits a single optical observation to identify with a line the direction of the object with respect to the ground station. The point of this oriented line that coincides with the target position is identified through the intersection with the orbital plane of the object obtained from the propagation of TLE parameters (see Figure 18).

The validity of the method has been checked taking as control values the ones obtained from the propagation of the first TLE released after the time of the observation. The algorithm elaborations of the data taken from MITO telescope and referred to the re-entry phase of Tiangong 1 are shown in Table 5 in comparison with the respective control values.

The algorithm results show in particular a mean shift of $1.525 \mathrm{Km}$ for the height and of $2.884 \mathrm{~km}$ for the range from the ground station compared to the values from the TLE propagation.

The good accuracy of the results obtained and the exiguous number of needed measures compared to a process of complete orbital determination, make this new tool capable of easily gain useful information in the ambit of the monitoring process of the phase of a space object orbital re-entry.

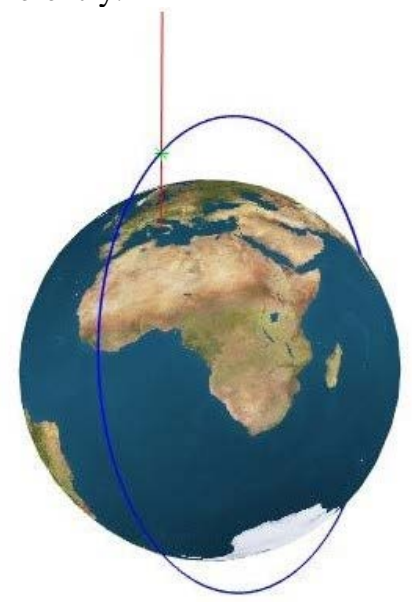

Figure 18. Intersection between the line connecting the ground station to the observed object position and the orbital plane

Table 5. Algorithm results of height and range compared to the values from TLE propagation 
$69^{\text {th }}$ International Astronautical Congress (IAC), Bremen, Germany, 1-5 October 2018.

Copyright (C2018 by the International Astronautical Federation (IAF). All rights reserved.

\begin{tabular}{|c|c|c|c|c|}
\hline DATE & $\begin{array}{c}\text { HEIGHT } \\
\text { (ALGORITHM) }\end{array}$ & $\begin{array}{c}\text { HEIGHT } \\
\text { (CONTROL } \\
\text { VALUE) }\end{array}$ & $\begin{array}{c}\text { RANGE FROM } \\
\text { MITO } \\
\text { (ALGORITHM) }\end{array}$ & $\begin{array}{l}\text { RANGE FROM } \\
\text { MITO } \\
\text { (CONTROL } \\
\text { VALUE) }\end{array}$ \\
\hline 03/07 18:38:00 & 252.42 & 251.42 & 561.16 & 559.6 \\
\hline 03/07 18:38:01 & 252.43 & 251.40 & 554.09 & 553.17 \\
\hline 03/07 18:38:31 & 251.98 & 251.00 & 374.97 & 374.49 \\
\hline 03/09 18:16:00 & 249.15 & 251.81 & 942.18 & 950.63 \\
\hline 03/09 18:16:01 & 249.12 & 251.8 & 934.60 & 943.66 \\
\hline 03/09 18:16:29 & 249.22 & 251.43 & 744.20 & 750.58 \\
\hline 03/09 18:16:30 & 249.20 & 251.42 & 736.85 & 743.78 \\
\hline 03/09 18:16:59 & 249.18 & 251.02 & 547.03 & 551.56 \\
\hline 03/09 18:17:00 & 249.17 & 251.00 & 540.05 & 545.16 \\
\hline 03/09 18:17:29 & 249.04 & 250.59 & 371.01 & 373.94 \\
\hline 03/09 18:17:30 & 249.03 & 250.57 & 365.36 & 368.77 \\
\hline 03/09 18:18:00 & 248.84 & 250.12 & 262.64 & 264.16 \\
\hline 03/10 18:05:30 & 249.98 & 250.98 & 730.10 & 731.37 \\
\hline 03/10 18:06:00 & 249.64 & 250.55 & 539.97 & 540.43 \\
\hline 03/10 18:06:01 & 249.55 & 250.54 & 533.30 & 534.42 \\
\hline 03/10 18:06:30 & 249.39 & 250.10 & 379.50 & 379.62 \\
\hline 02/15 04:59:00 & 265.50 & 261.46 & 285.03 & 280.26 \\
\hline 02/15 04:59:30 & 266.50 & 261.78 & 419.32 & 410.95 \\
\hline 02/15 05:00:01 & 268.35 & 262.09 & 615.20 & 601.35 \\
\hline 02/15 05:00:30 & 268.88 & 262.38 & 812.05 & 795.72 \\
\hline 02/15 05:00:32 & 267.56 & 262.40 & 822.21 & 809.41 \\
\hline 02/15 05:01:01 & 270.17 & 262.67 & 1031.92 & 1010.28 \\
\hline 02/16 04:53:59 & 258.99 & 259.90 & 279.19 & 280.40 \\
\hline 02/16 04:54:01 & 259.10 & 259.92 & 275.71 & 276.93 \\
\hline 02/19 04:39:29 & 257.34 & 255.65 & 699.21 & 696.30 \\
\hline 02/19 04:39:30 & 257.34 & 255.65 & 702.82 & 699.62 \\
\hline 02/19 04:40:07 & 257.84 & 255.87 & 856.90 & 857.33 \\
\hline 02/19 04:40:08 & 257.90 & 255.86 & 862.11 & 862.35 \\
\hline
\end{tabular}

\subsection{Lightcurve analysis}

A good technique used to determine the attitude of an orbital object is based on the study of the object's light curve derived information, i.e. the total reflected sunlight variation in time. This technique - derived from the asteroids attitude [2][3] - has recently been introduced in the uncooperative satellites optical attitude determination [4][5][6][7]. Indeed, the variation of the reflected light is a unique, intrinsic property of each object, since it depends by the object's shape, and by the material that compose it and of course, by its attitude. Thus, knowing the orbit and the geometry of the object is indeed possible to determine its attitude studying its light curves. The importance of knowing the attitude of an object resides in the fact that the attitude is one of the key parameters needed to obtain a precise orbit determination especially to compute the point of impact of a re-entry object as in the case of the Chinese space station Tiangong-1.

In the last years the S5Slab, of the Mechanical and Engineering Aerospace Department of the University of Rome La Sapienza, is developing and testing an algorithm able to find the attitude of an orbital object through its light curve [6]. The idea behind the method is to generate, for a selected target, different synthetic light curves with different attitude parameters, and compare them with the one observed. The synthetic light curve most similar to the observed one will be chosen as the presumable object's attitude.

To reach this goal, the first step was to develop a virtual reality simulation tool able to create as realistic as possible synthetic light curves in terms of optical and physical properties. To satisfy these requirements, the orbital position of the object will be recovered by propagating its orbit from the known TLE (Two-Line Element) through an SGP-4 (Simplified General Perturbations) routine. At the same time, the real positions of the observer and the Sun will be used to derive a precise value of the Phase angle Sun-objectobserver, aimed at obtaining an estimate of the light reflected by each object part. A realistic image of the object will be then produced at each instant of time using an advanced rendering algorithm, that takes into account parameters such as the atmospheric extinction, the object's shape and materials, and the direction of the sunlight. A particular effort is put to accurately reproduce the shadow areas cast among different 
object's components (see Figure 19). The second step was to develop an optimization algorithm, based on a genetic optimization algorithm, able to find the attitude of the object minimizing the residual between the real and the simulated light curves. In the specific case, for each generation thousand of different synthetic light are created, with different parameters. The six initial conditions $\left(\varphi_{0}, \theta_{0}, \psi_{0}, \mathrm{p}_{0}, \mathrm{q}_{0}, \mathrm{r}_{0}\right)$ in terms of Euler angles and angular velocity components, in the satellite reference frame, that give as result the light curve closer with the observed one will be used to populate the next generation and so on, till the residual between the synthetic light curves and the real one becomes minimum. Since the TLE and the SPG4 routing gives a prediction of the position of the satellite, in order to match with the real data a variable was introduced as a measure of synchronization time error between the predicted position of the satellite and the real one. Moreover, in the case of the Tiangong- 1 where the solar panels are able to move around an axis, an additional rotational parameter was added in the optimization to cope with that. Therefore, the genetic algorithm needs to move eight different parameters to find the solution: the three initial rotation angles, the three rotational velocities, the time shift and the rotational angle of the solar panel. The algorithm, after a validation on a synthetic dataset, was successfully used to determine a preamble asset of the Tiangong-1. In Figure 20 the synthetic light curve found by our algorithm in the case of the passage over Rome the 15/02/2018 is shown. The station seems to have: the $\mathrm{x}$-axis pointed to the velocity vector; the $y$-axis pointed to the Nadir with an angle between the solar panels and the Sun about $24^{\circ}$. This configuration create a drag area, in respect to the velocity vector, about $48 \mathrm{mq}$.

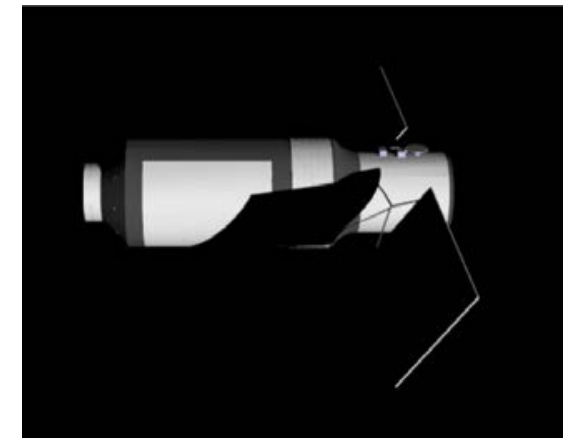

Figure 19. The figure shows the result of the rendering engine on the 3D model of the Tiangong- 1 . In the image are also depicted the shadow areas cast by the object on itself.

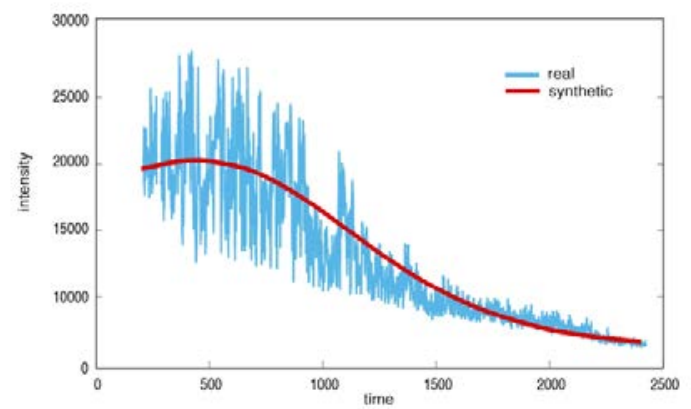

Figure 20. In figure in red the synthetic lightcurve found by the algorithm. In blue the real lightcurve of the Tiangog-1 passing over Rome the 15/02/2018.

\subsection{Re-entry predictions}

Launched on 29 September 2011, the first Chinese space station Tiangong-1 (COSPAR ID: 2011-053A; USSTRATCOM catalogue number: 37820) was originally intended for a controlled re-entry in the South Pacific Ocean Uninhabited Area (SPOUA) at the end of its planned operational lifetime in 2013. Nevertheless, the evolution of the average altitude of Tiangong-1 displayed regular orbital maintenance manoeuvres, executed between 2011 and 2013, followed by less frequent orbit raising manoeuvres during an extended mission phase, until December 2015 (Figure 21). Shortly afterwards, on 16 March 2016, the control of the space station was lost, due to an onboard fatal power failure, preventing a targeted re-entry of the spacecraft in the SPOUA.

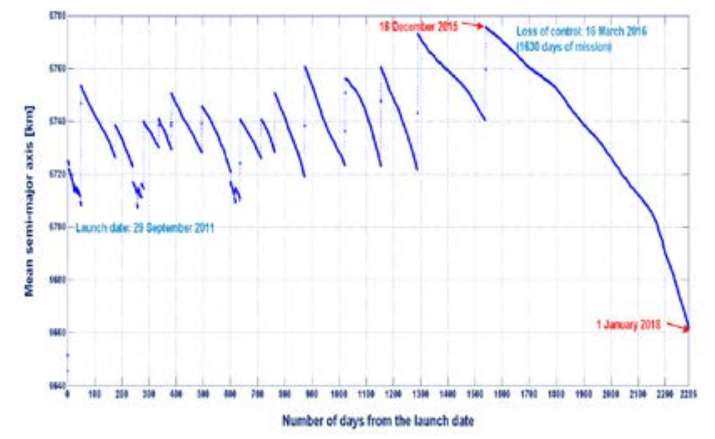

Figure 21. Evolution of the semi-major axis of Tiangong-1 since the launch, on 29 September 2011, in which the effects of the main manoeuvres are highlighted

Given its large size (a cylindrical body with a length of $10.4 \mathrm{~m}$ and an overall diameter of up to $3.4 \mathrm{~m}$, with two $7 \mathrm{~m} \times 3 \mathrm{~m}$ rectangular solar panels attached), the lift-off mass (8506 kg, including $1000 \mathrm{~kg}$ of propellant), and the characteristics of the materials, a complete demise of the space station upon re-entry would have been practically impossible. A not negligible fraction of the re-entering mass was expected to survive and hit the ground anywhere in between approximately 44 deg of 
latitude north and south. Moreover, a possible contamination on ground from the extremely toxic propellants still on board before re-entry (nearly $350 \mathrm{~kg}$ of mono-methyl hydrazine and nitrogen tetroxide, combined [8]) was not a priori excluded. For all of these reasons, even if no reliable information was available on the possible risk associated with the re-entry of Tiangong-1, the global casualty expectancy for this event was expected to overcome the internationally accepted threshold of $10^{-4}$ by at least 10 times.

Therefore, as in previous occasions in which the uncontrolled re-entry of an artificial space object had posed a potential threat to people and properties on the Italian territory, the Space Flight Dynamics Laboratory of CNR-ISTI carried out re-entry predictions and provided support to the Italian Space Agency (ASI) for the National Department of Civil Protection (DPC).

Although a few re-entry predictions had been already obtained at CNR-ISTI since the failure occurred in March 2016, when it was clear that the Chinese space station was no longer able to control its orbit, the reentry campaign started at the beginning of January 2018 and closed after the plunge of Tiangong-1 into the Earth’s atmosphere, on 2 April 2018.

The environmental conditions during the campaign were characterized by an extremely low solar activity, where the $10.7 \mathrm{~cm}$ solar flux $\left(\mathrm{F}_{10.7}\right)$, averaged over three solar rotations (81-day average $\mathrm{F}_{10.7}$ ), was around 70 solar flux units (sfu) $-1 \mathrm{sfu}=1 \times 10^{-22} \mathrm{Wm}^{-2} \mathrm{~Hz}^{-1}-$ all over the time span considered. The daily observed $\mathrm{F}_{10.7}$ was particularly stable during the last 1.5 months preceding the final decay of Tiangong-1 (Figure 22). There were instead numerous geomagnetic storms of class G1, and a single G2 storm, which significantly affected the orbit evolution of the space station, as it was in fact highlighted by the oscillations observed in the decay rate of its semi-major axis [9], [10]. It was found that in very low conditions of solar activity, as it was during the re-entry of Tiangong-1, also minor G1 storms could increase the local thermosphere density by more than $20 \%$ at the altitude of the Chinese space station. As a matter of fact, the G2 storm recorded during the night between 18 and 19 March 2018 moved earlier the nominal re-entry of Tiangong- 1 by nearly 31 hours.

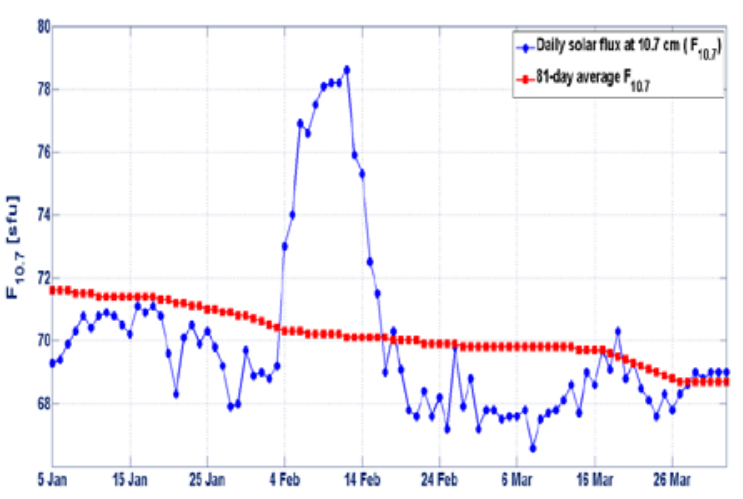

Figure 22. Daily and 81-day averaged solar flux at $10.7 \mathrm{~cm}$ during the re-entry campaign

Hundreds of re-entry predictions were performed since January 2018, using various combinations of atmospheric density models (NRLMSISE-00 and GOST2004) and time spans for the calibration of the ballistic parameter of Tiangong-1, in order to assess the sensitivity and the statistical distribution of the results obtained within a spectrum of reasonable hypotheses. From the beginning of February, the re-entry time began to converge towards the first week of April 2018 (Figure 23). But the G2 storm, occurred during the night of 18-19 March, caused a significant shift of the re-entry time, concentrating the re-entry predictions distribution from 30 March to 3 April 2018 (Figure 24). From 21 March 2018, the re-entry epoch stably settled on 1 April 2018. To be exact, in a 24-hour period between 01:00 UTC of 1 April and 01:00 UTC of 2 April. Within this 24-hour period, the only important shift occurred since 30 March, when an unusually long period of quiet geomagnetic conditions led to a cooling down of the atmosphere, with a consequent reduction of the density and a progressive extension of the residual lifetime of Tiangong-1.

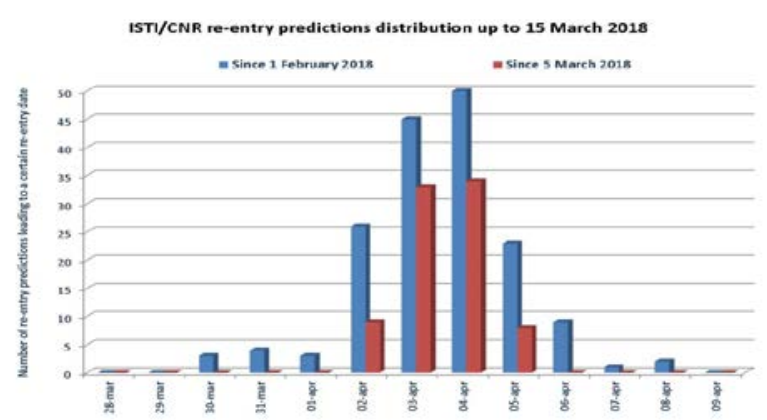

Figure 23. Distribution of the re-entry predictions from 1 February to 15 March 2018 


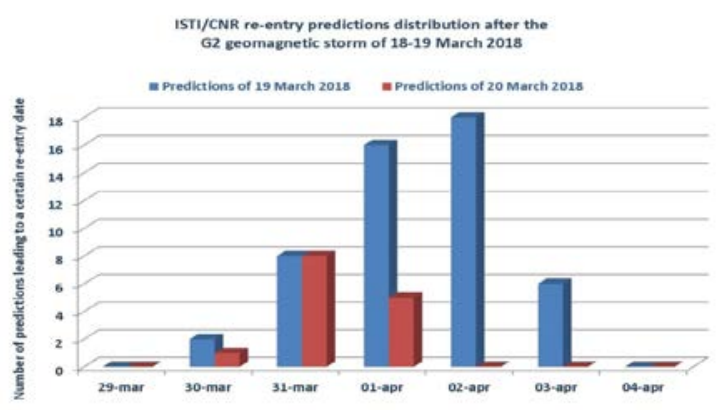

Figure 24. Distribution of the re-entry predictions carried out on 19 and 20 March 2018

The orbital evolution of uncontrolled spacecraft reentering the Earth's atmosphere from nearly circular orbits is mainly driven by the atmospheric drag. This implies that the uncertainties affecting the re-entry predictions are dominated by the modelling of the atmospheric drag, in turn characterized by inherent inaccuracies and biased related to: the physical properties and attitude of the spacecraft (its ballistic parameter); the atmospheric density model; the forecasts of the solar and geomagnetic activity influencing the atmospheric temperature, and the local air density as a consequence.

Therefore, in order to account for the possible uncertainties impinging upon the re-entry predictions, it is necessary to associate to each predicted re-entry epoch a so-called re-entry uncertainty window, inside which the actual re-entry might occur with a given confidence level.

The amplitude definition of a reliable and conservative uncertainty window has always been a very tricky task. There are no standard criteria to proceed, but a possible and reasonable approach is to take advantage of the expertise gained during a sufficiently high number of past re-entry events. With this in mind, the outcomes of a study carried out at CNR-ISTI, and based on twenty past re-entry exercises [11], were used to support the definition of the re-entry uncertainty windows for the campaign of Tiangong-1. On the basis of the statistical distribution of hundreds of re-entry predictions obtained in [11], it was found that an uncertainty window able to guarantee a confidence level of $90 \%$ should have assumed an amplitude of $\pm 20 \%$ around the computed nominal re-entry time. On the other hand, an uncertainty window amplitude of $\pm 30 \%$ would have been needed to achieve a confidence level of $95 \%$.

Therefore, considering that the re-entry uncertainty windows for Tiangong- 1 had to be used for civil protection applications, they were defined in order to guarantee a confidence level of at least 95\%. This means that the lower/upper limit of each re-entry uncertainty window was obtained by decreasing/increasing the computed residual lifetime of the spacecraft by $30 \%$. The re-entry uncertainty windows associated with the predicted re-entry time of Tiangong-1, since 21 March 2018, are shown in Figure 25. It is important to note that none of the re-entry uncertainty windows so obtained was violated by the post-event reference re-entry epoch, confirming the validity of the approach adopted.

The last re-entry prediction, with the relative uncertainty window (see Figure 26), was computed about nine hours before re-entry, and it was obtained using the last available US two-line element, referring to 16:07 UTC of 1 April 2018. According to this last prediction, the re-entry of Tiangong- 1 at $80 \mathrm{~km}$ of altitude would have occurred at 00:44 UTC of 2 April.

The re-entry uncertainty window had an amplitude of \pm 2.62 hours, centred on the nominal predicted reentry time. The orbit on which the re-entry of Tiangong1 actually occurred had already been identified nearly 18 hours before the final decay, but only the last uncertainty window allowed the exclusion of a re-entry over Italy with the targeted confidence level (> 95\%).

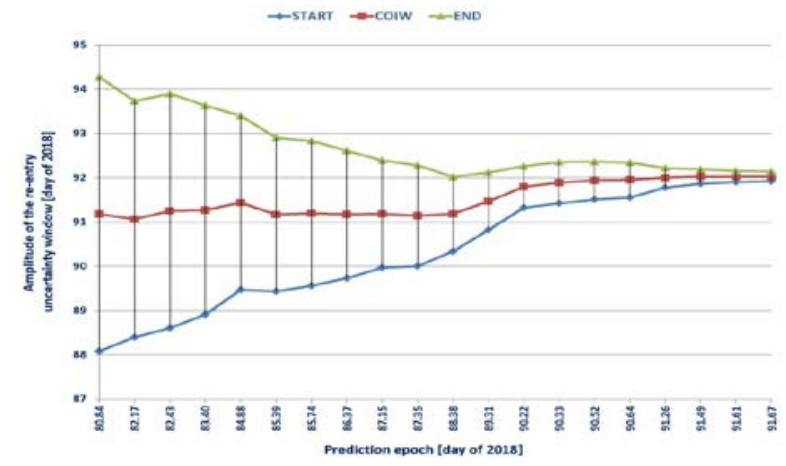

Figure 25. Uncertainty windows associated with the re-entry predictions carried out since 21 March 2018

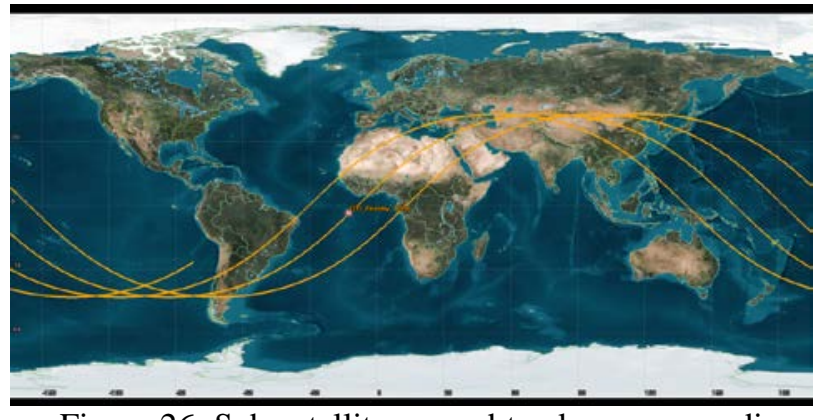

Figure 26. Sub-satellite ground tracks corresponding to the uncertainty window computed about 9 hours before re-entry

According to the post-event assessment released by the US Strategic Command, Tiangong-1 would have reached the altitude interface of $80 \mathrm{~km}$ at 00:10 UTC of 
2 April 2018, i.e. 33 minutes earlier than their last prediction issued about one hour before re-entry.

In addition to CNR-ISTI, ISOC also provided ASI with respective, autonomous object re-entry predictions.

ISOC predictions were based on the best possible estimation of apogee altitude, perigee altitude and reentry expected epoch, using the most recent orbital information, that could be either a TLE from JSpOC or an auto-generated object ephemeris, using national sensors data. Results were reported below, in Figure 27 and Figure 28.

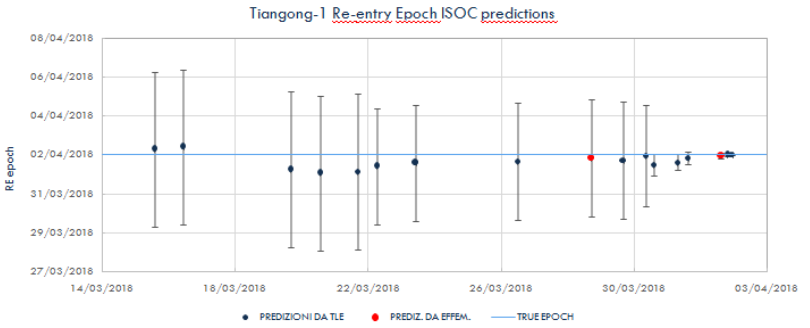

Figure 27. Tiangong-1 Re-entry Epoch ISOC Prediction Evolution
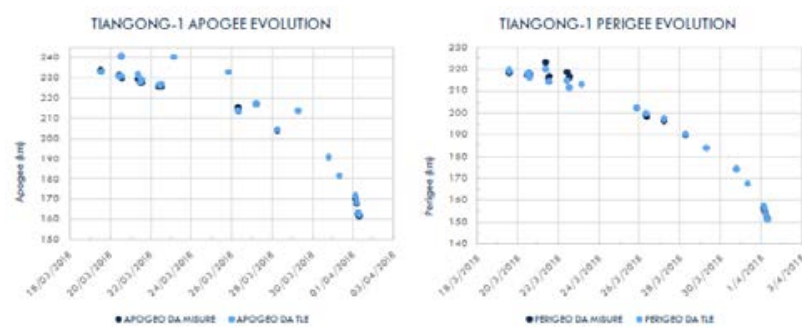

Figure 28. Tiangong-1 Apogee/Perigee ISOC Computation Evolution

\subsection{ASI role at Civil Protection Department}

The Italian Space Agency is the institutional point of contact and the interface with the Italian Civil Protection department for the re-entry campaigns of space objects. For the Tiangong- 1 case, as point of contact, ASI was also responsible of the deliverable of the products of the re-entry campaign, in particular the re-entry predictions provided by ISTI-CNR, conveniently arranged for the Civil Protection purposes.

A joint Tiangong-1 re-entry campaign was therefore organized involving research and governmental institutions. A joint technical board was timely set up and met regularly at the Italian Civil Protection headquarters for analysing the possible threats (e.g. critical infrastructures on the ground, air traffic control, safety procedures etc.). A timeline for managing operations was also agreed by identifying the most important milestones. Each milestone drove actions such as tasking different kind of sensors (e.g. optical vs radar) or evaluating the geographical distribution of the level of alert. The two most important events were marked by the time when the uncertainty prediction became less than 24 hours and when the actual re-entry ground tracks were identified. The committee became then permanently operational during the Tiangong-1 passages over Italy and ASI provided the technical information to the Civil Protection management in order to decide the activation/deactivation of the local departments spread across Italy.

ASI has been also coordinating with the Civil Protection for communicating with the media, which turned out to be a very sensitive task because of the widespread interest raised in the news by the Tiangong1 re-entry. The communication was of a high standard and resulted in conveying to the general public correct and timely information thus succeeding to prevent unjustified alarms.

\section{Conclusions}

Tiangong-1 re-entry has been an important test to understand the national SST capability in terms of both operations and procedures, and its responsiveness to high interest events. For the specific case of re-entry of uncontrolled objects, Italian SST system demonstrated to have all the necessary actors to monitor these events, providing information both to Government institutions and to general public. This experimental campaign highlighted also the operational capabilities acquired by the Italian SST team in terms of sensor tasking, data processing and product output and delivery.

International cooperation remains necessary in order to exchange information, especially in terms of TLE and observations, since the Italian distribution of sensors is mainly in the Italian territory and it does not permit to have a global coverage of the events, and to compare results, which can be not aligned for events difficult to be modelled and hence predicted.

\section{Acknowledgement}

The research activities and operations descripted in this paper have received funding from the European Commission Framework Programme H2020 and Copernicus, "SST - Space Surveillance and Tracking" contract No. 785257-2-3SST2016 and No. 237/G/GRO/COPE/16/8935-1SST2016.

We would like to thank the Institute of Space Sciences and Astronomy at the University of Malta for the development of the multibeam backend for BIRALES and for their contribution in the observation campaign.

\section{References}

[1] DECISION No 541/2014/EU OF THE EUROPEAN PARLIAMENT AND OF THE COUNCIL establishing a Framework for Space Surveillance and Tracking Support. L 158/227, Official Journal of European Union, 16 April 2014. 
[2] Minghe Shan, Jian Guo, and Eberhard Gill. Review and comparison of active space debris capturing and removal methods. Progress in Aerospace Sciences, 80:18-32, 2016

[3] Kjetil Wormnes, Ronan Le Letty, Leopold Summerer, Rogier Schonenborg, Olivier DuboisMatra, Eleonora Luraschi, Alexander Cropp, Holger Krag, and Jessica Delaval. Esa technologies for space debris remediation. In 6th European Conference on Space Debris, volume 1, pages 1-8. ESA Communications ESTEC, Noordwijk, The Netherlands, 2013.

[4] Ben Bradley and Penina Axelrad. Lightcurve inversion for shape estimation of geo objects from space-based sensors. In Univ. of Colorado. International Space Symposium for Flight Dynamics, 2014.

[5] C Fruh and Thomas Schildknecht. Analysis of observed and simulated light curves of space debris. In Proceedings of the international Astronautical Congress, 2010.

[6] Esther Linder, Jiri Silha, Thomas Schildknecht, and Monika Hager. Extraction of spin periods of space debris from optical light curves. 2015.
[7] Fabrizio Piergentili, Fabio Santoni, and Patrick Seitzer. Attitude determination of orbiting objects from lightcurve measurements. IEEE Transactions On Aerospace And Electronic Systems, 53(1):8190, 2017.

[8] C. Pardini, L. Anselmo, Estimate of Tiangong-1 Mass at Reentry, TG-1 ISTI/CNR Memo-01, Reentry Campaign IADC-2018-01, 19 March 2018.

[9] C. Pardini, L. Anselmo, The Uncontrolled Re-entry of Tiangong-1, Proceedings of the 36th IADC Plenary Meeting, IADC WG2 "Environment and Database”, Tsukuba International Congress Center, Tsukuba, Japan, 5-8 June 2018.

[10] C. Pardini, L. Anselmo, Uncontrolled Re-entry of Sizable Spacecraft and Rocket Bodies: A Potential Threat in the Airspace and on the Ground, Presentation PEDAS.1-0012-18, Space Debris Providing the Scientific Foundation for Action, 42nd COSPAR Scientific Assembly, Pasadena, CA, USA, 14-22 July 2018.

[11]C. Pardini, L. Anselmo, Assessing the risk and the uncertainty affecting the uncontrolled re-entry of manmade space objects, Journal of Space Safety Engineering 5 (2018) 46-62. 